\title{
A GENERALIZATION OF A THEOREM OF J. HOLUB
}

\author{
YURI ABRAMOVICH
}

(Communicated by John B. Conway)

\begin{abstract}
We present here a simple proof of the following result: Let $X$ be an arbitrary $C(K)$ or $L_{1}(\mu)$ space and let $T: X \rightarrow X$ be an arbitrary linear continuous operator. Then for at least one choice of signs.
\end{abstract}

$$
\|I \pm T\|=1+\|T\| .
$$

This is a slightly generalized version of a recent result due to $\mathbf{J}$. Holub [4].

In a recent work [4] J. Holub has proved the following surprising result: If $T$ is an arbitrary (continuous, linear) operator in the Banach space $C[0,1]$ of all continuous functions on the unit interval $[0,1]$, then for some $\gamma \in\{1,-1\}$

$$
\|I+\gamma T\|=1+\|T\| \text {. }
$$

In other words either $T$ or $-T$ or both satisfy the so-called Daugavet equation. We refer to $[2,6]$ for a history on the Daugavet equation and to $[2,4,5]$ for some related results. The aim of this article is to present a very simple proof of this (or rather, of a slightly more general) result, which allows one to understand its geometrical nature. We use the standard terminology and notations relating to Banach spaces, Banach lattices, and operators on them and we refer to [3] for all undefined terms.

Recall that a compact Hausdorff space $Q$ is said to be extremally disconnected if the closure of every open subset of $Q$ is again open.

Due to a well-known theorem of L. V. Kantorovich [3, Theorem 1.13] each $T \in L(C(Q), C(Q))$ has a modulus $|T| \in L(C(Q), C(Q))$ and, moreover, $\||T|\|=\|T\|$, where $L(X, X)$ denotes, as usual, the space of all operators on a Banach space $X$.

The key to our proof lies in making use of the following formula (due to the author [1], [3, Theorem 3.16]) for evaluating $|T|$. Namely, for an arbitrary $x \in C(Q)_{+}$,

$$
|T|(x)=\sup \{T e-T(x-e): e \in \mathscr{C}(x)\},
$$

where the supremum is taken over the set $\mathscr{C}(x)$ of all components of $x$. Recall that an element $e$ is a component of $x$ provided $e \wedge(x-e)=0$. In case $x=1$,

Received by the editors June 19, 1989.

1980 Mathematics Subject Classification (1985 Revision). Primary 47B38, 47A30; Secondary 46B25, 46E 15 . 
the constant 1 function, the set $\mathscr{C}(\mathbf{1})$ is simply the set of all characteristic functions $\chi_{A}$ of open and closed subsets $A$ of $Q$.

Theorem 1. Let $X$ be an arbitrary $C(K)$ or $L_{1}(\mu)$ space and let $T \in L(X, X)$. Then, for at least one $\gamma \in\{1,-1\}$,

$$
\|I+\gamma T\|=1+\|T\| \text {. }
$$

Proof. Let at first $X=C(Q)$, where $Q$ is an extremally disconnected compact Hausdorff space. It is plain that each positive operator $R: X \rightarrow X$ satisfies $\|R\|=\|R \mathbf{1}\|$ and thus

$$
\|T\|=\||T|\|=\||T|(\mathbf{1})\|=\sup \{(|T| \mathbf{1})(q): q \in Q\} .
$$

This implies that for each $\varepsilon>0$ there exists a $q_{0} \in Q$ and a closed and open neighborhood $V\left(q_{0}\right)$ of $q_{0}$ such that

$$
(|T| \mathbf{1})\left(q^{\prime}\right)>\|T\|-\varepsilon, \quad \forall q^{\prime} \in V\left(q_{0}\right) .
$$

In particular,

$$
|T| \mathbf{1}>f:=(\|T\|-\varepsilon) \chi_{V\left(q_{0}\right)}+(|T| \mathbf{1}) \chi_{Q \backslash V\left(q_{0}\right)} .
$$

Now, to evaluate $|T| \mathbf{1}$ we will apply (1):

$$
|T| \mathbf{1}=\sup \{T e-T(1-e): e \in \mathscr{C}(\mathbf{1})\} .
$$

In view of $\left(3^{\prime}\right)$ there exist $e \in \mathscr{C}(\mathbf{1})$ and $q \in V\left(q_{0}\right)$ such that

$$
(T e-T(\mathbf{1}-e))(q)>\|T\|-\varepsilon .
$$

If we put $x=e-(1-e)=2 e-1$, then (4) may be rewritten as

$$
(T x)(q)>\|T\|-\varepsilon \text {. }
$$

Obviously $|x|=1$ and thus, $x(q)=1$ or -1 . Let $x(q)=1$. Then

$$
\begin{aligned}
\|I+T\| & \geq\|(I+T)(x)\|=\|x+T x\| \\
& \geq x(q)+(T x)(q)=1+(T x)(q) \\
& \geq 1+\|T\|-\varepsilon .
\end{aligned}
$$

If $x(q)=-1$, then

$$
\|-I+T\| \geq-x(q)+(T x)(q) \geq 1+\|T\|-\varepsilon .
$$

Since $\varepsilon$ is arbitrary, (6) and (5) imply (2) in the special case under consideration.

The general case follows immediately from this one. Indeed, if $X$ is an $L_{1}(\mu)$ space, then $X^{*}$ is a $C(Q)$ space with an extremally disconnected $Q$ and hence (for an appropriate sign)

$$
\|I \pm T\|=\left\|I^{*} \pm T^{*}\right\|=1+\left\|T^{*}\right\|=1+\|T\| .
$$

Finally, if $X$ is an arbitrary $C(K)$ space, then $X^{*}$ is an $L_{1}(\mu)$ space and again we can go to the conjugate operator. 
Remarks. 1. The above idea of the proof can be briefly expressed as follows: For a given $\varepsilon>0$, we can find an $x \in C(Q)$ and a $q \in Q$ such that $|x(q)|=$ $\|x\|=1$ and $|T x(q)| \geq\|T\|-\varepsilon$. Obviously this implies (2).

2. The same proof remains valid for an arbitrary $A M$-space and, thus, the following is true:

Theorem 2. Let $X$ be an arbitrary $A L$ - or $A M$-space and let $T \in L(X, X)$. Then either $\|I+T\|$ or $\|I-T\|$ equals $1+\|T\|$.

3. A similar approach based on the Banach lattice theory makes it possible to obtain a simple proof of the results connected with Holub's generalizations of the Daugavet theorem to weakly compact operators. These results will appear elsewhere [2]. This is just the case when the Daugavet equation is valid for both signs. We mention incidentally that another proof of Theorem 2, a proof based on the theory of orthomorphisms, has also been found by K. D. Schmidt.

Added in proof. I am thankful to Professor A. T. Wickstead for informing me that prior to the work of $\mathrm{J}$. Holub the same property of operators was also discovered by J. Duncan, C. M. McGregor, J. D. Price, and A. J. White in the article "The numerical index of a normed space" [J. London Math. Soc. (2) 2 (1970), 481-488.]

\section{ACKNOWLEDGMENTS}

The author would like to express his thanks to Professors C. D. Aliprantis and C. Foias for their interest in this work, and to the referee for his helpful comments.

\section{REFERENCES}

1. Y. A. Abramovich, Injective envelopes of normed lattices, Soviet Math. Dokl. 12 (1971), 511 514.

2. Y. A. Abramovich and K. Schmidt, Daugavet's equation and orthomorphisms, preprint.

3. C. D. Aliprantis and O. Burkinshaw, Positive operators, Academic Press, New York, London, 1985.

4. J. Holub, A property of weakly compact operators on $C[0,1]$, Proc. Amer. Math. Soc. 97 (1986), 396-398.

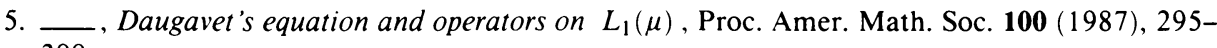
300.

6. A. R. Sourour, MR $88 \mathrm{j}$, no. 47037.

Department of Mathematics, Indiana University-PuRdue University at Indianapolis, 1125 EASt 38Th StREeT, INDIANAPOLIS, INDIANa 46205 